EXTENDED REPORT

\title{
Threshold Amsler grid as a screening tool for asymptomatic patients on hydroxychloroquine therapy
}

\author{
A Almony, S Garg, R K Peters, R Mamet, J Tsong, B Shibuya, R Kitridou, A A Sadun
}

Br J Ophthalmol 2005;89:569-574. doi: 10.1136/bjo.2004.050120

See end of article for authors' affiliations ......................

Correspondence to: Alfredo A Sadun, $M D, P h D$, Department of Ophthalmology, Keck School of Medicine, University of Southern California, Doheny Eye Institute, 1450 San Pablo Street, Los Angeles, CA 90033-1026, USA; asadun@usc.edu

Accepted for publication 2 November 2004

\begin{abstract}
Background/aims: Patients taking hydroxychloroquine (HCQ) are at risk of developing classic bull's eye maculopathy. Currently, the standard Amsler grid (AG) is one of the most useful methods to identify such lesions. However, AG is a suprathreshold target and may not detect relative central scotomas. The aim of this study was to determine if the threshold Amsler grid (TAG) test, which varies light transmission through two cross polarising filters, allows increased detection of scotomas caused by HCQ toxicity.

Methods: 56 rheumatological patients taking $\mathrm{HCQ}$ and 12 similar patients not taking $\mathrm{HCQ}$ were tested by $A G$, red Amsler grid (RAG), and TAG.

Results: No scotomas were observed in patients never treated with HCQ. Among patients who had been treated with HCQ, AG revealed scotomas in two of $56(3.64 \%)$ patients; in contrast, six $(10.7 \%)$ and 37 $(66.1 \%)$ scotomas were identified by RAG and TAG testing respectively. Additionally, the average area of each scotoma detected by all three methods expanded from 34.5 square degrees of central field loss on AG testing to 71 square degrees on RAG and 117 on TAG.

Conclusion: By decreasing the perceived luminance of the suprathreshold AG, TAG testing provides a novel alternative to detect shallow scotomas and areas of depressed retinal activity secondary to $\mathrm{HCQ}$ toxicity.
\end{abstract}

$\mathrm{T}$ he 4-aminoquinolone compounds, chloroquine and hydroxychloroquine sulfate (HCQ), have long been used to treat connective tissue disorders, most specifically rheumatoid arthritis (RA) and systemic lupus erythematosus (SLE). Currently, HCQ, which is far less toxic than chloroquine, continues to be used as an antimalarial drug and in the treatment of connective tissue diseases worldwide. Amsler grid (AG) testing has been a standard means of assessing the central visual field since its introduction in 1947 and a valuable screening method in order to prevent the sometimes irreversible manifestations of ocular toxicity. However, AG provides a suprathreshold target to examine the central visual field and relative scotomas may go undetected before they become absolute scotomas. In contradistinction, threshold Amsler grid (TAG) varies perceived luminance by implementing the use of cross polarising filters, thereby decreasing the image contrast and serving as a more sensitive test to detect relative scotomas and depressions in patients with maculopathy. ${ }^{1-3}$ (fig 1)

The aim of this study was to determine if TAG testing allows early and increased sensitivity in the detection of scotomas caused by HCQ toxicity.

\section{PATIENTS AND METHODS}

Upon receiving institutional review board (IRB) approval for our study, we recruited patients from the rheumatology outpatient clinic at LA County USC in Los Angeles and obtained informed consent from them before study enrolment. All 77 individuals who were approached agreed to participate in this study.

The inclusion criteria were consecutive patients seen from 18 June 2002 to 25 July 2002 in the LA County-USC rheumatology outpatient clinic with a known history of connective tissue disorder and a best corrected visual acuity of at least 20/50. Patients with other disorders that might produce a maculopathy including diabetes mellitus, a history of ocular trauma, or other ocular disorders were excluded retrospectively $(n=7)$. Patients on other medications that

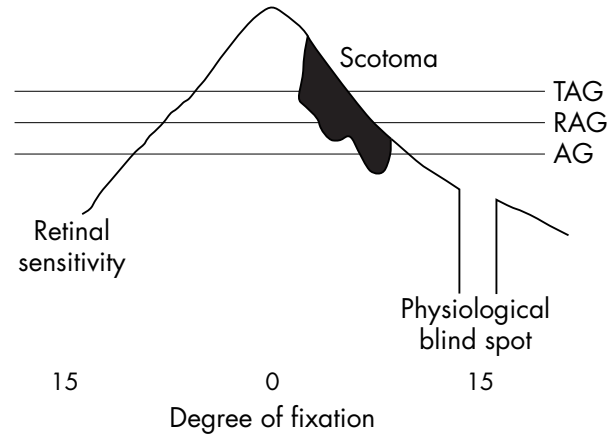

Figure 1 A schematic of Tranquair's analogy "an island of vision in a sea of blindness" displays the essential elements behind TAG testing. Reducing contrast reduces the stimulus; this is analogous to cutting through the island near its highest point. The standard AG cuts the lower part of the island where retinal sensitivity is low, while the TAG cuts the island where retinal sensitivity is higher. Relative scotoma is represented by the black area. Suprathreshold standard Amsler grid (AG), red Amsler grid (RAG), and threshold Amsler grid (TAG).

might produce retinal toxicity including digoxin were also excluded $(n=1)$. One patient did not complete the study because she developed a headache during the examination. Of the remaining 68 patients, $56(82.4 \%)$ were taking HCQ for their connective tissue disorder. The 12 patients who were not taking HCQ had never taken it and served as controls.

Data were collected from the patients' medical charts and corroborated with the patient in a written questionnaire regarding date of birth, sex, ethnicity, diagnosis of connective tissue disorder, HCQ dose and duration, current medications, history of digoxin use, medical and surgical history, ocular history, and baseline and follow up ophthalmological

Abbreviations: $A G$, Amsler grid; $H C Q$, hydroxychloroquine; RA, rheumatoid arthritis; RAG, red Amsler grid; SLE, systemic lupus erythematosus; TAG, threshold Amsler grid 
examinations (patients at LAC-USC are referred for a complete baseline ophthalmological examination before starting HCQ therapy which includes a dilated retina examination and central visual field sensitivity testing by an Amsler grid as well as complete annual ophthalmological follow up examinations). In the questionnaire, patients were also asked to report any visual complaints. Each patient was weighed during the examination.

For all patients, each eye was tested separately using five examinations while the untested eye remained occluded. Each patient received an ophthalmological examination that included near visual acuity using the Snellen chart, colour vision using pseudo-isochromatic plates Nos 1-8, and AG testing (white grid on black background as described by Amsler ${ }^{4}{ }^{5}$ ). These examinations are the standard in screening for HCQ toxicity. ${ }^{67}$ RAG (red grid on black background) is used by some clinicians and was included in the examination. ${ }^{89}$ We also added TAG as the final component of the examination. For TAG testing, patients viewed the standard white on black AG through specialised cross polarising filters (fig 2) to reduce the perceived luminance by changing the amount of light that goes through the specialised glasses. The angle of polarisation between the two filters in the glasses was increased by 1 degree at a time until the patient stated that (s)he could no longer see the grid but could still see the white central dot. The angle was then decreased by approximately 2 degrees to increase the luminance, making the grid barely visible. Patients were asked to focus on the central white dot and report any abnormalities, which they drew on the Amsler recording chart paper (fig 3). If necessary, the polarised lenses were placed over reading glasses.

Patient demographics are shown in table 1. RA, SLE, and other connective tissue disorders affect all ethnic groups, however, the majority of our population at LA County-USC is Hispanic and this was reflected in both the control and HCQ groups equally. Most (64 of 68 or $94.1 \%$ ) were women (the prevalence of RA is approximately 2.5 times, and of SLE 9-10 times higher in women than in $\operatorname{men}^{10}$ ) and of Hispanic ethnicity ( 52 of 68 or $76.5 \%$ ). Patients in both groups were also essentially the same average age and range of ages. The

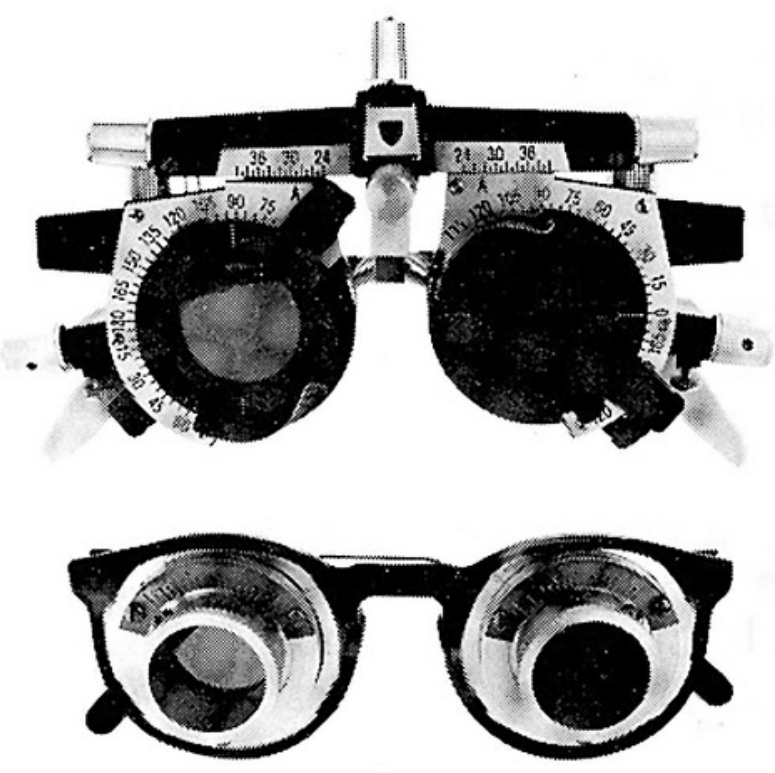

Figure 2 Threshold Amsler grid lenses containing pairs of polarised filters in each ocular. As the front filter is rotated, light transmission is reduced.

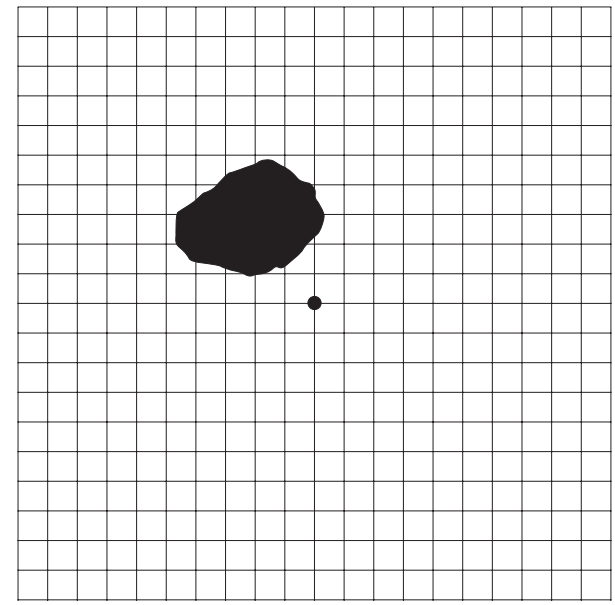

Figure 3 Example of results of threshold Amsler grid testing on a patient identified with a central scotoma.

mean age of the 68 patients was 43 years (range 2166 years).

\section{RESULTS}

Patient visual examination data are shown in table 2. As expected, no central scotomas were detected in any patients not taking HCQ. Most of our subjects were women, therefore our findings may not be generalisable to men.

Among the 56 patients that took HCQ for at least 1 month, there was no correlation between scotoma detection and sex, ethnicity, diagnosis, HCQ dose, or length of HCQ therapy. From our analysis, when looking at high and low risk based on milligrams HCQ per kilogram patient weight, the data did not associate patients on higher doses of HCQ with an increased number of scotoma detection. In addition, as expected, there was no bias toward scotoma detection in the left or right eye. Finally, as mentioned previously, patients with known ocular disease were excluded from the study. Data collected from the patients' ocular examinations did not reveal any abnormal findings in the baseline or annual dilated fundus examination. Humphrey 10-2 visual field testing had not been performed on any patients and baseline and follow up Amsler grid testing had been normal.

Scotomas were identified by AG in two of 56 patients taking HCQ (3.6\%). In contrast, RAG identified scotomas in five of 56 patients $(8.9 \%)$ and TAG identified at least one scotoma in 25 (almost $45 \%$ ) of 56 patients taking HCQ. TAG testing detected both patients identified as having one or more scotoma by AG, and all five of the patients identified as having at least one scotoma by RAG. The average area of the two scotomas identified by AG were 34.5 mean square degrees per scotoma, compared to an average area of 71.2 mean square degrees per scotoma for the six detected by RAG, and 117 mean square degrees per scotoma for the 37 scotomas identified by TAG. The same five scotomas were reported to be 70 mean square degrees per scotoma when identified by RAG, and 272.4 mean square degrees when identified by TAG. Finally, TAG was more likely than RAG and AG to pick up scotomas in both eyes of a patient versus only one eye (nearly $25 \%$ more than RAG and $50 \%$ more than AG).

\section{DISCUSSION}

Previous studies have defined the diagnosis of HCQ retinopathy using two different methods. Bernstein defined it as the development of persistent paracentral or central visual field scotomas to suprathreshold white stimulus. ${ }^{11}$ According 


\begin{tabular}{|c|c|c|c|c|c|c|c|c|c|}
\hline Subject & Age & Weight (kg) & Diagnosis & Sex & Ethnicity & HCQ & $\begin{array}{l}\text { Dose } \\
\text { (mg/kg/day) }\end{array}$ & $\begin{array}{l}\text { No of months } \\
\text { on plaquenil }\end{array}$ & Continuous dose \\
\hline 1 & 38 & 145.5 & Other & $\mathrm{F}$ & AA & $\mathrm{N}$ & & & \\
\hline 2 & 26 & 65.5 & Other & $\mathrm{F}$ & $\mathrm{H}$ & $\mathrm{N}$ & & & \\
\hline 3 & 58 & 69.5 & Other & $\mathrm{F}$ & H & $N$ & & & \\
\hline 4 & 64 & 85.9 & Other & $M$ & $\mathrm{H}$ & $\mathrm{N}$ & & & \\
\hline 5 & 41 & 99.5 & Other & $M$ & W & $\mathrm{N}$ & & & \\
\hline 6 & 60 & 53.6 & RA & $\mathrm{F}$ & $\mathrm{H}$ & $\mathrm{N}$ & & & \\
\hline 7 & 54 & 55.9 & RA & $\mathrm{F}$ & H & $\mathrm{N}$ & & & \\
\hline 8 & 58 & 62.7 & Other & $\mathrm{F}$ & H & $\mathrm{N}$ & & & \\
\hline 9 & 33 & 40.9 & RA & $\mathrm{F}$ & H & $\mathrm{N}$ & & & \\
\hline 10 & 46 & 88.6 & SLE & $\mathrm{F}$ & H & $\mathrm{N}$ & & & \\
\hline 11 & 42 & 56.4 & Other & $\mathrm{F}$ & H & $\mathrm{N}$ & & & \\
\hline 12 & 40 & 66.8 & RA & M & A & $\mathrm{N}$ & & & \\
\hline 13 & 44 & 65.0 & RA & $\mathrm{F}$ & H & Y & 6.2 & 24 & Y \\
\hline 14 & 26 & 76.4 & SLE & $\mathrm{F}$ & H & Y & 5.2 & 96 & Y \\
\hline 15 & 57 & 50.0 & RA & $\mathrm{F}$ & H & Y & 8.0 & 18 & Y \\
\hline 16 & 47 & 68.6 & RA & $\mathrm{F}$ & $\mathrm{H}$ & Y & 5.8 & 7 & $\mathrm{Y}$ \\
\hline 17 & 52 & 100.0 & Other & M & W & Y & 2.4 & 11 & $\mathrm{~N}$ \\
\hline 18 & 48 & 72.7 & RA & $\mathrm{F}$ & $\mathrm{H}$ & Y & 5.5 & 24 & Y \\
\hline 19 & 43 & 73.6 & SLE & $\mathrm{F}$ & H & Y & 5.4 & 43 & Y \\
\hline 20 & 48 & 69.5 & RA & $\mathrm{F}$ & H & Y & 5.8 & 36 & Y \\
\hline 21 & 46 & 44.5 & SLE & $\mathrm{F}$ & A & Y & 6.7 & 72 & Y \\
\hline 22 & 43 & 71.4 & SLE & $\mathrm{F}$ & $\mathrm{H}$ & Y & 5.6 & 156 & Y \\
\hline 23 & 64 & 45.5 & RA & $\mathrm{F}$ & A & Y & 8.8 & 6 & Y \\
\hline 24 & 42 & 96.8 & SLE & $\mathrm{F}$ & AA & Y & 4.1 & 72 & $\mathrm{~N}$ \\
\hline 25 & 46 & 62.3 & $\mathrm{RA}$ & $\mathrm{F}$ & $\mathrm{H}$ & Y & 6.4 & 13 & Y \\
\hline 26 & 53 & 70.9 & RA & $\mathrm{F}$ & H & Y & 5.6 & 72 & Y \\
\hline 27 & 62 & 87.3 & SLE & $\mathrm{F}$ & $\mathrm{H}$ & Y & 4.6 & 120 & $Y$ \\
\hline 28 & 45 & 82.7 & Other & $\mathrm{F}$ & $\mathrm{AA}$ & Y & 4.8 & 72 & $Y$ \\
\hline 29 & 55 & 67.7 & RA & $\mathrm{F}$ & $\mathrm{H}$ & Y & 5.9 & 96 & $Y$ \\
\hline 30 & 59 & 60.0 & SLE & $\mathrm{F}$ & $\mathrm{H}$ & Y & 6.7 & 36 & $Y$ \\
\hline 31 & 27 & 59.1 & SLE & $\mathrm{F}$ & W & Y & 6.8 & 36 & Y \\
\hline 32 & 60 & 55.5 & RA & $\mathrm{F}$ & $\mathrm{H}$ & Y & 5.4 & 7 & $Y$ \\
\hline 33 & 35 & 64.1 & RA & $\mathrm{F}$ & H & Y & 6.2 & 24 & Y \\
\hline 34 & 42 & 77.3 & SLE & $\mathrm{F}$ & AA & Y & 5.2 & 240 & $Y$ \\
\hline 35 & 66 & 55.9 & RA & $\mathrm{F}$ & A & Y & 3.6 & 132 & $\mathrm{~N}$ \\
\hline 36 & 53 & 54.5 & RA & $\mathrm{F}$ & AA & Y & 7.3 & 36 & $Y$ \\
\hline 37 & 24 & 85.9 & SLE & $\mathrm{F}$ & $\mathrm{H}$ & Y & 4.7 & 48 & Y \\
\hline 38 & 24 & 66.4 & RA & $\mathrm{F}$ & $\mathrm{H}$ & Y & 6.0 & 6 & $Y$ \\
\hline 39 & 21 & 121.4 & SLE & $\mathrm{F}$ & H & Y & 3.3 & 48 & Y \\
\hline 40 & 34 & 59.1 & RA & $\mathrm{F}$ & $\mathrm{H}$ & Y & 6.8 & 24 & $Y$ \\
\hline 41 & 21 & 65.5 & SLE & $\mathrm{F}$ & H & Y & 6.1 & 12 & Y \\
\hline 42 & 41 & 65.5 & RA & $\mathrm{F}$ & $\mathrm{H}$ & Y & 6.1 & 5 & $Y$ \\
\hline 43 & 34 & 61.4 & SLE & $\mathrm{F}$ & H & Y & 6.5 & 72 & Y \\
\hline 44 & 32 & 75.5 & Other & $\mathrm{F}$ & $\mathrm{H}$ & Y & 5.3 & 24 & $Y$ \\
\hline 45 & 49 & 89.5 & $\mathrm{RA} / \mathrm{SLE}$ & $\mathrm{F}$ & AA & Y & 3.4 & 27 & $Y$ \\
\hline 46 & 52 & 87.3 & Other & $\mathrm{F}$ & $\mathrm{H}$ & Y & 4.6 & 48 & $Y$ \\
\hline 47 & 56 & 61.8 & RA & $\mathrm{F}$ & $\mathrm{H}$ & Y & 6.5 & 126 & $\mathrm{~N}$ \\
\hline 48 & 34 & 60.5 & RA & $\mathrm{F}$ & $\mathrm{H}$ & Y & 6.6 & 48 & $Y$ \\
\hline 49 & 28 & 64.1 & RA & $\mathrm{F}$ & $\mathrm{H}$ & $Y$ & 6.2 & 24 & $Y$ \\
\hline 50 & 27 & 74.1 & Other & $\mathrm{F}$ & $\mathrm{H}$ & Y & 5.4 & 36 & $Y$ \\
\hline 51 & 32 & 60.9 & SLE & $\mathrm{F}$ & $\mathrm{H}$ & Y & 6.6 & 120 & $Y$ \\
\hline 52 & 49 & 65.9 & RA & $\mathrm{F}$ & $\mathrm{H}$ & Y & 6.1 & 8 & $Y$ \\
\hline 53 & 29 & 61.8 & SLE & $\mathrm{F}$ & $\mathrm{H}$ & Y & 4.9 & 180 & $Y$ \\
\hline 54 & 35 & 102.3 & RA & $\mathrm{F}$ & $\mathrm{H}$ & Y & 3.9 & 12 & $Y$ \\
\hline 55 & 38 & 58.2 & RA & $\mathrm{F}$ & $\mathrm{H}$ & Y & 6.9 & 4 & $Y$ \\
\hline 56 & 30 & 61.8 & SLE & $\mathrm{F}$ & $\mathrm{H}$ & Y & 6.5 & 12 & $Y$ \\
\hline 57 & 42 & 84.1 & RA & $\mathrm{F}$ & $\mathrm{H}$ & Y & 2.4 & 36 & $Y$ \\
\hline 58 & 34 & 86.4 & SLE & $\mathrm{F}$ & $\mathrm{H}$ & $Y$ & 4.6 & 36 & $Y$ \\
\hline 59 & 23 & 40.9 & SLE & $\mathrm{F}$ & A & $Y$ & 6.1 & 48 & $\mathrm{~N}$ \\
\hline 60 & 37 & 67.7 & Other & $\mathrm{F}$ & $\mathrm{H}$ & $Y$ & 5.9 & 6 & $Y$ \\
\hline 61 & 50 & 76.4 & SLE & $\mathrm{F}$ & $\mathrm{H}$ & Y & 5.2 & 36 & $Y$ \\
\hline 62 & 53 & 59.5 & RA & $\mathrm{F}$ & H & Y & 6.7 & 72 & Y \\
\hline 63 & 52 & 54.5 & RA & $\mathrm{F}$ & H & Y & 7.3 & 1 & Y \\
\hline 64 & 60 & 61.8 & RA & $\mathrm{F}$ & H & Y & 6.5 & 36 & Y \\
\hline 65 & 53 & 68.6 & RA & $\mathrm{F}$ & H & Y & 5.8 & 60 & $Y$ \\
\hline 66 & 25 & 48.2 & Other & $\mathrm{F}$ & A & Y & 4.2 & 36 & $\mathrm{~N}$ \\
\hline 67 & 26 & 61.4 & RA & $\mathrm{F}$ & H & Y & 6.5 & 12 & $\mathrm{~N}$ \\
\hline 68 & 48 & 61.4 & SLE & $\mathrm{F}$ & $\mathrm{AA}$ & Y & 6.5 & 12 & $\mathrm{~N}$ \\
\hline
\end{tabular}

to Easterbrook, HCQ retinopathy is defined as the presence of reproducible, bilateral field defects detected by two different visual field tests. ${ }^{12}$ More specifically, these tests are the Amsler grid and an automated, 10 degree visual field test. In both definitions, the finding of permanent functional visual loss is essential to the diagnosis. For the purposes of our study, a gold standard for HCQ toxicity was not defined. Our aim was to determine if there was a significant difference in relative scotoma detection using methods-AG, RAG, and TAG testing - which would be quick, easy, and reproducible in a clinical setting and at home in patients without previous ocular diagnosis related to HCQ toxicity or otherwise. A 


\begin{tabular}{|c|c|c|c|c|c|c|c|}
\hline Subject & HCQ & Eye & VA & Colour test plates & $\mathbf{A G}^{*}$ & $\mathrm{RAG}^{*}$ & TAG* \\
\hline \multirow[t]{2}{*}{1} & $\mathrm{~N}$ & RE & $20 / 20$ & $8 / 8$ & & & \\
\hline & & LE & $20 / 20$ & $8 / 8$ & & & \\
\hline \multirow[t]{2}{*}{2} & $\mathrm{~N}$ & RE & $20 / 30$ & $7 / 8$ & & & \\
\hline & & LE & $20 / 30$ & $8 / 8$ & & & \\
\hline \multirow[t]{2}{*}{3} & $\mathrm{~N}$ & RE & $20 / 40$ & $8 / 8$ & & & \\
\hline & & LE & $20 / 30$ & $8 / 8$ & & & \\
\hline \multirow[t]{2}{*}{4} & $\mathrm{~N}$ & RE & $20 / 25$ & $8 / 8$ & & & \\
\hline & & LE & $20 / 30$ & $8 / 8$ & & & \\
\hline \multirow[t]{2}{*}{5} & $\mathrm{~N}$ & RE & $20 / 25$ & $8 / 8$ & & & \\
\hline & & LE & $20 / 20$ & $8 / 8$ & & & \\
\hline 6 & $\mathrm{~N}$ & RE & $20 / 25$ & $7 / 8$ & & & \\
\hline & & LE & $20 / 25$ & $8 / 8$ & & & \\
\hline 7 & $\mathrm{~N}$ & RE & $20 / 30$ & $8 / 8$ & & & \\
\hline & & LE & $20 / 25$ & $8 / 8$ & & & \\
\hline 8 & $\mathrm{~N}$ & RE & $20 / 40$ & $8 / 8$ & & & \\
\hline & & LE & $20 / 30$ & $7 / 8$ & & & \\
\hline 9 & $\mathrm{~N}$ & RE & $20 / 25$ & $7 / 8$ & & & \\
\hline & & LE & $20 / 25$ & $8 / 8$ & & & \\
\hline 10 & $\mathrm{~N}$ & RE & $20 / 40$ & $8 / 8$ & & & \\
\hline & & LE & $20 / 50$ & $8 / 8$ & & & \\
\hline 11 & $\mathrm{~N}$ & RE & $20 / 25$ & $8 / 8$ & & & \\
\hline & & LE & $20 / 25$ & $8 / 8$ & & & \\
\hline 12 & $\mathrm{~N}$ & RE & $20 / 30$ & $8 / 8$ & & & \\
\hline & & LE & $20 / 30$ & $8 / 8$ & & & \\
\hline 13 & Y & RE & $20 / 20$ & $8 / 8$ & & & \\
\hline & & LE & $20 / 20$ & $8 / 8$ & & & \\
\hline 14 & Y & RE & $20 / 20$ & $8 / 8$ & & & \\
\hline & & LE & $20 / 20$ & $8 / 8$ & & & \\
\hline 15 & Y & RE & $20 / 25$ & $8 / 8$ & & & \\
\hline & & LE & $20 / 25$ & $8 / 8$ & & & \\
\hline 16 & Y & RE & $20 / 30$ & $7 / 8$ & & & \\
\hline & & LE & $20 / 40$ & $8 / 8$ & & & \\
\hline 17 & Y & RE & $20 / 25$ & $8 / 8$ & & & \\
\hline & & LE & $20 / 25$ & $8 / 8$ & & & \\
\hline 18 & Y & RE & $20 / 30$ & $7 / 8$ & & & 140 \\
\hline & & LE & $20 / 30$ & $7 / 8$ & & & 29 \\
\hline 19 & Y & RE & $20 / 30$ & $8 / 8$ & & & \\
\hline & & LE & $20 / 30$ & $8 / 8$ & & & \\
\hline 20 & Y & RE & $20 / 25$ & $8 / 8$ & & & \\
\hline & & LE & $20 / 30$ & $8 / 8$ & & & \\
\hline 21 & Y & RE & $20 / 30$ & $8 / 8$ & & & \\
\hline & & LE & $20 / 30$ & $8 / 8$ & & & \\
\hline 22 & Y & RE & $20 / 30$ & $8 / 8$ & & 78 & 71 \\
\hline & & LE & $20 / 25$ & $8 / 8$ & & 77 & \\
\hline 23 & Y & RE & $20 / 30$ & $8 / 8$ & & & \\
\hline & & LE & $20 / 30$ & $8 / 8$ & & & \\
\hline 24 & Y & RE & $20 / 25$ & $8 / 8$ & & & \\
\hline & & LE & $20 / 25$ & $8 / 8$ & & & \\
\hline 25 & Y & RE & $20 / 25$ & $8 / 8$ & & & 113 \\
\hline & & LE & $20 / 25$ & $8 / 8$ & & & \\
\hline 26 & Y & RE & $20 / 40$ & $8 / 8$ & & & \\
\hline & & LE & $20 / 40$ & $8 / 8$ & & & \\
\hline 27 & Y & RE & $20 / 30$ & $8 / 8$ & & & \\
\hline & & LE & $20 / 25$ & $8 / 8$ & & & \\
\hline 28 & Y & RE & $20 / 25$ & $8 / 8$ & & & 76 \\
\hline & & LE & $20 / 30$ & $8 / 8$ & & & \\
\hline 29 & Y & RE & $20 / 30$ & $8 / 8$ & & & \\
\hline & & LE & $20 / 30$ & $8 / 8$ & & & \\
\hline 30 & Y & RE & $20 / 30$ & $8 / 8$ & & & \\
\hline & & LE & $20 / 30$ & $8 / 8$ & & & \\
\hline 31 & Y & RE & $20 / 30$ & $8 / 8$ & 62 & 48 & 261 \\
\hline & & LE & $20 / 25$ & $8 / 8$ & & & \\
\hline 32 & Y & RE & $20 / 40$ & $7 / 8$ & & & 42 \\
\hline & & LE & $20 / 20$ & $7 / 8$ & & & \\
\hline 33 & Y & RE & $20 / 30$ & $8 / 8$ & & & \\
\hline & & LE & $20 / 30$ & $8 / 8$ & & & \\
\hline 34 & Y & RE & $20 / 30$ & $8 / 8$ & & & \\
\hline & & LE & $20 / 30$ & $8 / 8$ & & & \\
\hline 35 & Y & RE & $20 / 40$ & $8 / 8$ & & & \\
\hline & & LE & $20 / 40$ & $8 / 8$ & & & \\
\hline 36 & Y & RE & $20 / 50$ & $8 / 8$ & & & \\
\hline & & LE & $20 / 50$ & $8 / 8$ & & & 100 \\
\hline 37 & Y & RE & $20 / 20$ & $7 / 8$ & & & \\
\hline & & LE & $20 / 20$ & $8 / 8$ & & & \\
\hline 38 & Y & RE & $20 / 20$ & $8 / 8$ & & & \\
\hline & & LE & $20 / 20$ & $8 / 8$ & & & \\
\hline 39 & Y & RE & $20 / 30$ & $8 / 8$ & & & \\
\hline & & LE & $20 / 30$ & $8 / 8$ & & & 160 \\
\hline 40 & Y & RE & $20 / 40$ & $8 / 8$ & & & 49 \\
\hline & & LE & $20 / 30$ & $8 / 8$ & & & \\
\hline
\end{tabular}




\begin{tabular}{|c|c|c|c|c|c|c|c|}
\hline Subject & $\mathrm{HCQ}$ & Eye & VA & Colour test plates & $\mathbf{A G}^{*}$ & RAG* $^{*}$ & TAG $^{*}$ \\
\hline \multirow[t]{2}{*}{41} & Y & RE & $20 / 20$ & $8 / 8$ & & & \\
\hline & & LE & $20 / 20$ & $8 / 8$ & & & \\
\hline \multirow[t]{2}{*}{42} & Y & RE & $20 / 40$ & $8 / 8$ & & & 35 \\
\hline & & LE & $20 / 30$ & $8 / 8$ & 7 & & 49 \\
\hline \multirow[t]{2}{*}{43} & Y & RE & $20 / 20$ & $8 / 8$ & & & 77 \\
\hline & & LE & $20 / 20$ & $8 / 8$ & & & \\
\hline \multirow[t]{2}{*}{44} & Y & RE & $20 / 20$ & $8 / 8$ & & & 120 \\
\hline & & LE & $20 / 20$ & $8 / 8$ & & & \\
\hline \multirow[t]{2}{*}{45} & Y & RE & $20 / 30$ & $8 / 8$ & & & \\
\hline & & LE & $20 / 30$ & $8 / 8$ & & & \\
\hline \multirow[t]{2}{*}{46} & Y & RE & $20 / 30$ & $7 / 8$ & & 100 & 385 \\
\hline & & LE & $20 / 40$ & $7 / 8$ & & & 340 \\
\hline \multirow[t]{2}{*}{47} & Y & RE & $20 / 40$ & $8 / 8$ & & & 180 \\
\hline & & LE & $20 / 30$ & $8 / 8$ & & & 180 \\
\hline \multirow[t]{2}{*}{48} & Y & RE & $20 / 20$ & $7 / 8$ & & & 74 \\
\hline & & LE & $20 / 20$ & $7 / 8$ & & & \\
\hline \multirow[t]{2}{*}{49} & Y & RE & $20 / 25$ & $8 / 8$ & & & \\
\hline & & LE & $20 / 20$ & $8 / 8$ & & & \\
\hline \multirow[t]{2}{*}{50} & Y & RE & $20 / 20$ & $8 / 8$ & & & \\
\hline & & LE & $20 / 20$ & $8 / 8$ & & & \\
\hline \multirow[t]{2}{*}{51} & Y & RE & $20 / 25$ & $8 / 8$ & & & 28 \\
\hline & & LE & $20 / 25$ & $8 / 8$ & & & 67 \\
\hline \multirow[t]{2}{*}{52} & Y & $\mathrm{RE}$ & $20 / 30$ & $8 / 8$ & & & 11 \\
\hline & & LE & $20 / 30$ & $8 / 8$ & & & 11 \\
\hline \multirow[t]{2}{*}{53} & Y & RE & $20 / 30$ & $8 / 8$ & & & \\
\hline & & LE & $20 / 30$ & $8 / 8$ & & & 71 \\
\hline \multirow[t]{2}{*}{54} & Y & RE & $20 / 25$ & $8 / 8$ & & 24 & 272 \\
\hline & & LE & $20 / 20$ & $8 / 8$ & & & 128 \\
\hline \multirow[t]{2}{*}{55} & Y & RE & $20 / 20$ & $8 / 8$ & & & \\
\hline & & LE & $20 / 20$ & $8 / 8$ & & & \\
\hline \multirow[t]{2}{*}{56} & Y & RE & $20 / 25$ & $7 / 8$ & & & \\
\hline & & LE & $20 / 25$ & $7 / 8$ & & & \\
\hline \multirow[t]{2}{*}{57} & Y & RE & $20 / 25$ & $8 / 8$ & & & 70 \\
\hline & & LE & $20 / 25$ & $8 / 8$ & & & 84 \\
\hline 58 & Y & RE & $20 / 25$ & $8 / 8$ & & & \\
\hline & & LE & $20 / 20$ & $8 / 8$ & & & \\
\hline 59 & Y & RE & $20 / 20$ & $8 / 8$ & & & \\
\hline & & $\mathrm{LE}$ & $20 / 20$ & $8 / 8$ & & & 120 \\
\hline 60 & $Y$ & RE & $20 / 30$ & $8 / 8$ & & & \\
\hline & & LE & $20 / 30$ & $8 / 8$ & & & \\
\hline 61 & Y & RE & $20 / 40$ & $8 / 8$ & & & \\
\hline & & $\mathrm{LE}$ & $20 / 30$ & $8 / 8$ & & & \\
\hline 62 & Y & RE & $20 / 25$ & $8 / 8$ & & & \\
\hline & & LE & $20 / 20$ & $8 / 8$ & & & \\
\hline 63 & Y & RE & $20 / 25$ & $8 / 8$ & & 100 & 373 \\
\hline & & $\mathrm{LE}$ & $20 / 25$ & $8 / 8$ & & & 375 \\
\hline 64 & $Y$ & RE & $20 / 30$ & $8 / 8$ & & & \\
\hline & & $\mathrm{LE}$ & $20 / 30$ & $8 / 8$ & & & \\
\hline 65 & $Y$ & RE & $20 / 30$ & $8 / 8$ & & & 24 \\
\hline & & $\mathrm{LE}$ & $20 / 30$ & $8 / 8$ & & & 120 \\
\hline 66 & $Y$ & RE & $20 / 20$ & $8 / 8$ & & & 9 \\
\hline & & $\mathrm{LE}$ & $20 / 20$ & $8 / 8$ & & & 9 \\
\hline 67 & Y & RE & $20 / 20$ & $8 / 8$ & & & \\
\hline & & LE & $20 / 20$ & $8 / 8$ & & & \\
\hline 68 & Y & RE & $20 / 25$ & $\begin{array}{l}8 / 8 \\
8 / 8\end{array}$ & & & $\begin{array}{l}40 \\
40\end{array}$ \\
\hline & & LE & $20 / 25$ & $8 / 8$ & & & 40 \\
\hline
\end{tabular}

follow up study will be necessary to determine true HCQ toxicity.

Both control patients and those with a history of HCQ therapy occasionally reported that they could not see one or more corners of the grid during AG, RAG, and/or TAG testing. Missing corners of the grid have also been reported by normal subjects. ${ }^{1}$ Loss of corners may not necessarily reflect pathology since the grid corners are further from the fovea and hence retinal sensitivity is decreased in that area. Therefore, these data were not considered in the analysis. One or more scotomas were identified in two patients using AG testing; five by RAG and 25 patients by TAG testing. Therefore, TAG was 12.5 times as likely to identify a patient with scotoma compared to AG and five times as likely as RAG $(p=0.003)$. Both patients identified as having one or more scotoma by AG were also identified by
TAG and all five of the patients identified as having one or more scotomas by RAG were also identified by TAG, leading us to believe that TAG affords greater sensitivity than AG or RAG. Of the 38 scotomas detected by one or more of the three methods of testing, AG detected 5\% while TAG detected $97 \%$.

Our data demonstrated that nearly $45 \%$ of the patients taking HCQ appeared to have one or more scotomas by TAG. While $45 \%$ seems high, a high false positive rate is unlikely in that no patients in the control group were identified as having any scotomas. Retinal toxicity has been reported internationally in up to $28 \%$ of patients taking HCQ and the American Academy of Ophthalomolgy (AAO) recommendations on HCQ screening indicate that patients may develop relative scotomas which are asymptomatic and do not show up on the fundus examination. ${ }^{13}$ 
The mean area of the two scotomas identified by AG were 34.5 mean square degrees per scotoma, compared to 71.2 square degrees per scotoma for the six detected by RAG, and 117 square degrees per scotoma for the 37 scotomas identified by TAG. TAG identified an affected area more than three times the area of that identified by AG. Of the five scotomas detected by both RAG and TAG, TAG detected an average area per scotoma nearly four times that of RAG $(p=0.02)$. TAG tests the central visual field at threshold and is able to detect a larger affected area of the same scotoma because it includes relative scotomas of lesser severity.

It seems most reasonable to assume that an appropriate screening test would detect scotomas bilaterally. In our study, AG never detected the involvement of both eyes while RAG only detected it $20 \%$ of the time. TAG identified one or more scotomas in both eyes at least $52 \%$ of the time.

One individual, patient 63, had been on HCQ treatment for only 1 month but had the largest bilateral scotomas in terms of area of any patient in our study. She had never taken HCQ previously and did not have a history of ocular pathology, but had missed her appointment for a baseline ophthalmological examination. It is highly unlikely that the TAG results were secondary to HCQ toxicity, therefore, this case highlights the need to obtain both a baseline and an early examination in patients who are starting HCQ treatment.

TAG is an easy to perform, quick, and high yield alternative to detect shallow/relative scotomas reflecting areas of depressed retinal activity secondary to HCQ toxicity. These scotomas may go undetected early on with AG or RAG testing and lead to irreversible visual changes. TAG offers clinicians a novel alternative for the detection of central visual field defects in patients receiving HCQ therapy. TAG can also be used as a quantitative method to monitor the progression of macular changes in these patients.

If retinal toxicity is diagnosed, the HCQ is usually immediately discontinued as this may lead to clinical improvement. ${ }^{14}$ Reversibility of visual loss diminishes with time, therefore, early detection is important. ${ }^{15-17}$ Some of the retinal changes seen in our patient population may be reversible functional changes. Because no gold standard for toxicity was defined, and because HCQ therapy is such an important component of treatment in many patients with connective tissue disorders, our study cannot be used to determine when HCQ therapy should be stopped. None the less, while controversy does exist in the literature regarding the validity and sensitivity of AG and TAG testing, the results from our study show significant findings with TAG testing that may be indicative of permanent retinal toxicity. ${ }^{18}{ }^{19}$ Long term follow up of HCQ patients with many retinal measures will help to determine if the scotomas have enlarged or changed and when the point of irreversibility is crossed. In the meantime, prudence requires careful monitoring by the most sensitive measures including TAG.

In recognition of the great benefit that HCQ brings to patients with rheumatoid arthritis and other connective tissue disorders, we recommend careful monitoring and only consideration of drug cessation with progression of TAG scotomas or demonstration of classic ophthalmological impairments. Owing to the serious nature of the retinal toxicity that can be caused by HCQ, even after cessation of the drug, TAG can be used as an effective screening method to detect these scotomas before permanent damage ensues.

\section{ACKNOWLEDGEMENTS}

We are grateful to Robert A Almony, III, Lori Levin, Michael Payle, Michelle Rios, Toufan Rahimpour, Anne Rosenfeld, Fred RossCisneros, and NIH EY03040.

\section{Authors' affiliations}

A Almony, S Garg, A A Sadun, Doheny Eye Institute, Los Angeles, CA, USA

R K Peters, R Mamet, Department of Preventive Medicine, Keck School of Medicine of the University of Southern California, Los Angeles, CA, USA

J Tsong, Department of Ophthalmology, George Washington University Medical Center, Washington, DC, USA

B Shibuya, R Kitridou, Department of Rheumatology, Keck School of Medicine of the University of Southern California, Los Angeles, CA, USA

\section{REFERENCES}

1 Wall M, Sadun AA. Threshold Amsler grid testing: cross-polarizing lenses enhance yield. Arch Ophthalmol 1986;104:520-3.

2 Wall M, Collins C, May DR. Low-intensity grids improve sensitivity of Amsler grid testing in diabetic patients without background retinopathy. Ann Ophthalmol 1990;22:96-100.

3 Saadati HG, Sadun AA. Three-dimensional quantification of central visual field defects using the modified threshold Amsler grid test. Presented at Association for Research in Vision and Ophthalmology, Fort Lauderdale, FL May, 1997.

4 Amsler M. L'examen qualitif de la fonction maculaire. Ophthalmologica $1947 ; 114: 248-61$.

5 Amsler M. Quantitative and qualitative vision. Trans Ophthalmol Soc UK 1949;69:397-410.

6 Easterbrook M. Detection and prevention of maculopathy associated with anti-malarial agents. Int Ophthalmol Clin 1999;39:49-57.

7 Ruiz RS, Saatci OA. Chloroquine and hydroxychloroquine retinopathy: how to follow affected patients. Ann Ophthalmol 1991;23:290-1.

8 Fielder A, Graham E, Jones S, et al. Royal College of Ophthalmologists guidelines: ocular toxicity and hydroxychloroquine. Eye 1998;12:907-9.

9 Grierson DJ. Hydroxychloroquine and visual screening in a rheumatology outpatient clinic. Ann Rheum Dis 1997;56:188-90.

10 Klippel JH, Stone JH, Crofford L. Primer on the rheumatic diseases. 12th ed. Arthritis Foundation, 2001

11 Bernstein HN. Ocular safety of hydroxychloroquine. South Med J 1992;85:274-9.

12 Easterbrook M. The ocular safety of hydroxychloroquine. Semin Arth Rhem 1993;23(Suppl 1):62-7.

13 Marmor MF, Carr RE, Easterbrook M, et al. Recommendations on screening for chloroquine and hydroxychloroquine retinopathy. Ophthalmology 2002; 109:1377-82.

14 Crew SJ. Chloroquine retinopathy with recovery in early stages. Lancet 1964;ii:436-8.

15 Ehrenfeld M, Nesher R, Merin S. Delayed onset of chloroquine retinopathy. Br J Ophthalmol 1986;70:281-3

16 Sassasni JW, Brucker AJ, Cobbs W, et al. Progressive chloroquine retinopathy. Ann Ophthalmol 1983;15:19-22.

17 Bernstein HN. Ophthalmological considerations and testing in patients receiving long-term antimalarial therapy. Am J Med 1983;75(Suppl):25-34.

18 Ariyasu RG, Lee PP, Linton KP, et al. Sensitivity, specificity, and predictive values of screening tests for eye conditions in a clinic-based population. Ophthalmology 1996;103:1751-60.

19 Schuchard RA. Validity and interpretation of Amsler grid reports. Arch Ophthalmol 1993;111:776-8. 